\title{
Optimum Lattice Functions For Electron Cooling
}

\author{
J. Bosser, C. Carli, M. Chanel, S. Maury, D. Moel, G. Tranquille
}

\begin{abstract}
Cooling down time measurements carried out at LEAR with 4.2 MeV/nucleon $\mathrm{Pb}^{54+}$ ions and various combinations of lattice functions led to unexpected results. Notably, a finite dispersion was found to decrease strongly the horizontal cooling down times. Furthermore, intermediate betatron functions yielded shorter cooling down times than large ones and an increase of the cooler length by a factor 2 did not lead to the expected increase in the cooling rate. The aim of this report is to explain, at least qualitatively, the experimental observations.
\end{abstract}

Presented at COOLO3

International Workshop on Beam Cooling and Related Topics

May 19 - 23, 2003

Hotel Mt. Fuji , Lake Yamanaka, Japan 


\title{
Optimum Lattice Functions for Electron Cooling
}

\author{
J. Bosser, C. Carli, M. Chanel, S. Maury, D. Möhl, G. Tranquille \\ CERN, Geneva, Switzerland
}

\begin{abstract}
Cooling down time measurements carried out at LEAR with $4.2 \mathrm{MeV} /$ nucleon $\mathrm{Pb}^{54+}$ ions and various combinations of lattice functions led to unexpected results. Notably, a finite dispersion was found to decrease strongly the horizontal cooling down times. Furthermore, intermediate betatron functions yielded shorter cooling down times than large ones and an increase of the cooler length by a factor 2 did not lead to the expected increase in the cooling rate. The aim of this report is to explain, at least qualitatively, the experimental observations.
\end{abstract}

Key words:

\section{Introduction}

It has been proposed to provide the ion beam needed for LHC by accumulation with strong electron cooling(1). In order to validate the principle and to optimize parameters, experimental investigations have been carried out between 1994 and $1997(2)$ with $\mathrm{Pb}^{54+}$ ions at $4.2 \mathrm{MeV} /$ nucleon at the LEAR machine. Amongst the tests performed were extensive cooling rate measurements with different electron cooling currents and various lattice parameters at the location of the electron cooler. The experimental observations were rather different from expectations. They are summarized in section 2. In section 3, mechanisms explaining qualitatively the difference between initial expectations and observations, are given.

\section{Experimental Observations}

Extensive measurements of the cooling down times have been carried out with various electron cooler currents and lattice parameters at the location of the

Preprint submitted to Elsevier Science 11 July 2003 


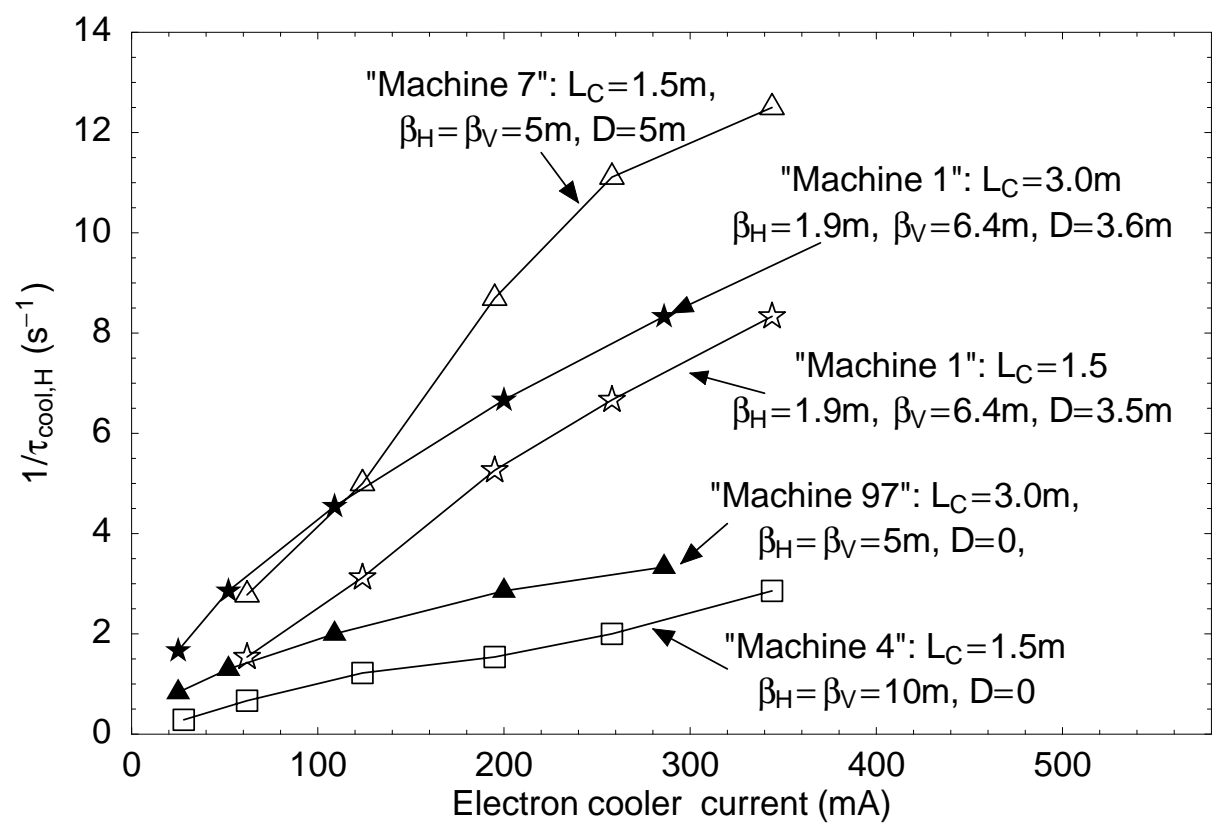

Fig. 1. Inverse of the horizontal cooling down times versus electron cooler current for different lattice functions and with different cooler lengths at the LEAR machine (circumference $25 \pi \mathrm{m}$ ).

cooler. Here, by cooling down times, we denote the time to cool the beam from an initial physical emittance of $40 \mu \mathrm{m}$ down to $4 \mu \mathrm{m}$ (the emittance being defined as $(2 \sigma)^{2} / \beta_{T}$, with $\sigma$ the RMS beam width and, $\beta_{T}$ the Twiss betatron function). For every setting, the cooling rate has been optimized by adjusting the ion orbit. The inverse of measured horizontal cooling down times $\tau_{c o o l, H}$ are plotted in Fig. 1 versus cooler current and for lattices obtained by different settings of the lattice quadrupoles.

The following observations drawn from Fig. 1 are in contradiction to initial expectations :

- Beneficial effect of a finite dispersion :

Fastest cooling has been achieved with the settings "machine 7" and "machine 1", which have both a relatively large dispersion. From theoretical considerations, the dispersion was expected to play only a minor role. In similar measurements with $50 \mathrm{MeV}$ proton beams $(3 ; 5)$, the beneficial effect of a finite dispersion has been found as well.

- Slow cooling with large betatron functions :

For large betatron function the typical transverse velocities (in a co-moving coordinate system) of the ion beam, given by $\beta \gamma c \sqrt{\epsilon / \beta_{T}}$, are well inside the velocity distribution of the electron beam. Here, $\beta$ and $\gamma$ are the relativistic coefficients, $c$ the velocity of light $\epsilon$ the emittance of the beam and $\beta_{T}$ the Twiss betatron function. One expects fast electron cooling in this regime, where the force experienced by the ions depends linearly on their velocity. 
"Machine 4" has large betatron functions at the location of the cooler, but contrary to expectations, cooling rates measured with this setting were small.

- Dependence of the cooling rate on the cooler length :

For the measurements carried out in 1997, the length of the cooler (where ions and electrons are co-moving) has been doubled from $1.5 \mathrm{~m}$ to $3 \mathrm{~m}$ in order to test the expected increase in cooling rate by a factor 2. From Fig. 1, one sees that for "machine 1" this increase in cooling rate was observed only for small currents. In fact, with the longer electron cooler, the dependance of the cooling rate on the current was not linear any more, in contradiction to expectations. For completeness, it should be mentioned that, to increase the cooler length, two shorter solenoids were combined and a dip of the field at the centre has been corrected by adding an correcting coil. Thus, the quality of the magnetic field has suffered due the increase of the cooler length.

\section{Qualitative Explanation of the Observations}

A key ingredient to explain the observations reported in section 2 is the parabolic velocity profile of the electron beam. The space charge density of the electron beam itself creates a potential depending on the radial position. Since the total energy of the electrons does not depend on the position, the longitudinal velocity depends on the radial position. The relative momentum offset of the electrons as a function of the distance $r$ from the center is given by :

$$
\frac{\Delta p}{p}=\frac{I e}{4 \pi \epsilon_{0} \beta^{3} \gamma c E_{0}} \frac{r^{2}}{a^{2}}
$$

with $I$ the cooler current, $a=2.5 \mathrm{~cm}$ the radius of the electron beam, $\gamma$ and $\beta$ the relativistic factors and, $e$ and $E_{0}$ the electron charge and rest mass. For a moderate cooler current of $200 \mathrm{~mA}$, this relative momentum shift of the electrons at the border of the electron beam amounts to $\Delta p / p \approx 14 \cdot 10^{-3}$.

\subsection{Beneficial effect of a finite dispersion}

At least two different mechanisms can explain why the cooling rates increase with a finite dispersion. Both are given here. To determine which effect dominates, precise simulations are necessary, which in turn are only possible with realistic models for the friction force experienced by the ions in the cooler. 


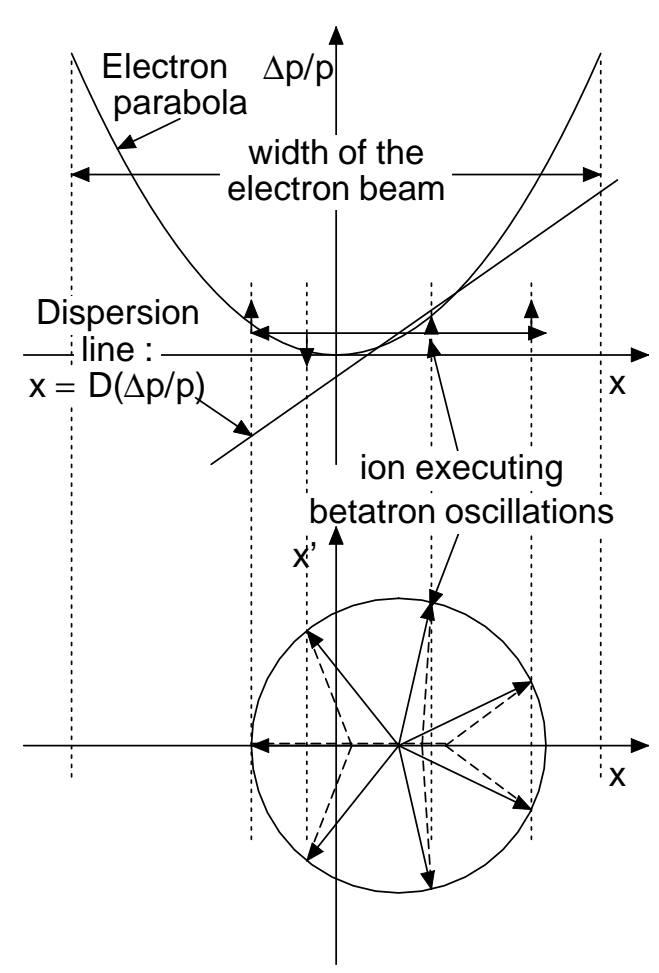

Fig. 2. Dispersive cooling

\subsubsection{Dispersive Cooling}

The concept outlined in this section is very similar to the dispersive cooling reported in (4). The principle is best explained with the help of Fig. 2. One ion executing betatron oscillations is shown in phase space (lower image) and in a plot of longitudinal velocity versus position. Depending on the betatron phase, the ion will be accelerated or decelerated. The change in momentum leads to a change in closed orbit proportional to the dispersion and, thus, to a change of the betatron oscillation vector. The betatron oscillation vectors before (solid arrows) and after passage of the cooler (dashed arrows) are plotted in the phase space plot. For most phases, the betatron oscillation amplitude is reduced and, thus, transverse cooling takes place due to the longitudinal cooling force.

\subsubsection{Increase of transverse cooling rate due to better overlap with the elec- tron parabola}

Another explanation is based on the idea that with a finite dispersion the ion velocity-position distribution can better overlap with the electron beam parabola $(2 ; 3 ; 5)$. It is outlined in Fig. 3. The upper image Fig. 3a shows the region covered by the ion beam and the electron beam profile for zero dispersion. The ions have relatively large longitudinal velocity offset w.r.t. the electrons. Thus, the transverse cooling force is reduced and the cooling times increase. With a finite dispersion, the overlap between ion beam and electrons 


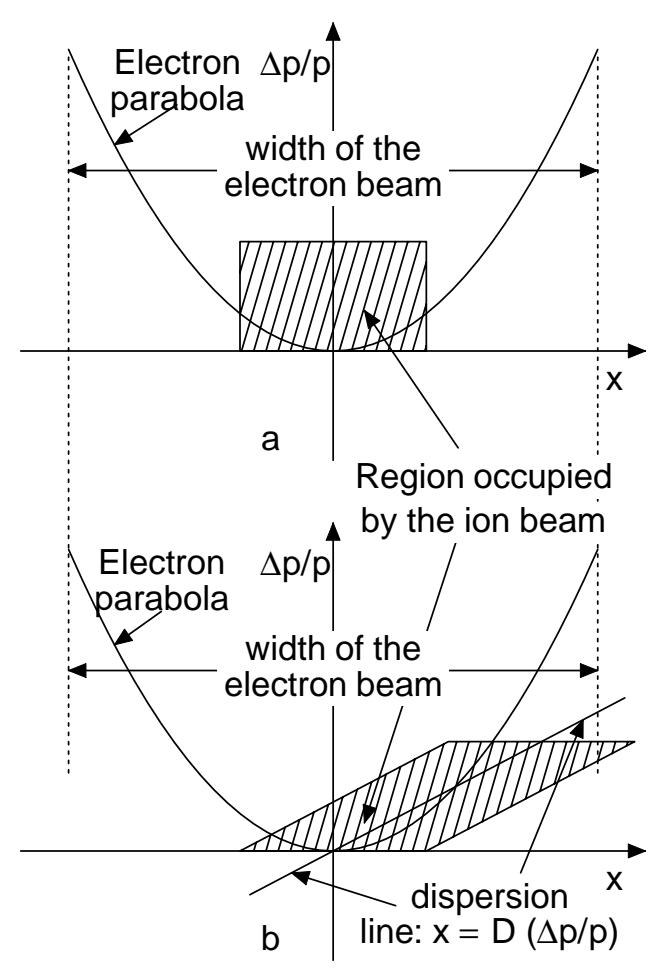

Fig. 3. Improved overlap between the ion beam and the parabolic electron velocity profile.

can be improved as indicated in Fig. 3b. This mechanism might explain the observed beneficial effect of a finite dispersion to achieve fast electron cooling.

\subsection{Slow Cooling with large Betatron Functions}

Fig. 4 shows the regions, occupied by an ion beam and by the electron beam (parabola), in a diagram showing longitudinal velocity versus horizontal position $x$. Both images are for zero dispersion, but for different betatron functions (and the same emittances). Fig. 4a is the situation for a large betatron function. For most betatron phases, the longitudinal velocity of the ions is far from the electron parabola and thus the transverse friction force is reduced and the cooling rate decreased. If in addition the ion beam becomes larger than the electron beam, the cooling rates are further decreased. On the other hand, as depicted in Fig. 4b, the overlap between the ion beam and the electron parabola may be improved by reducing the betatron function at the location of the cooler. This in turn may increase the horizontal cooling force experienced by the ion and thus lead to shorter cooling times. 


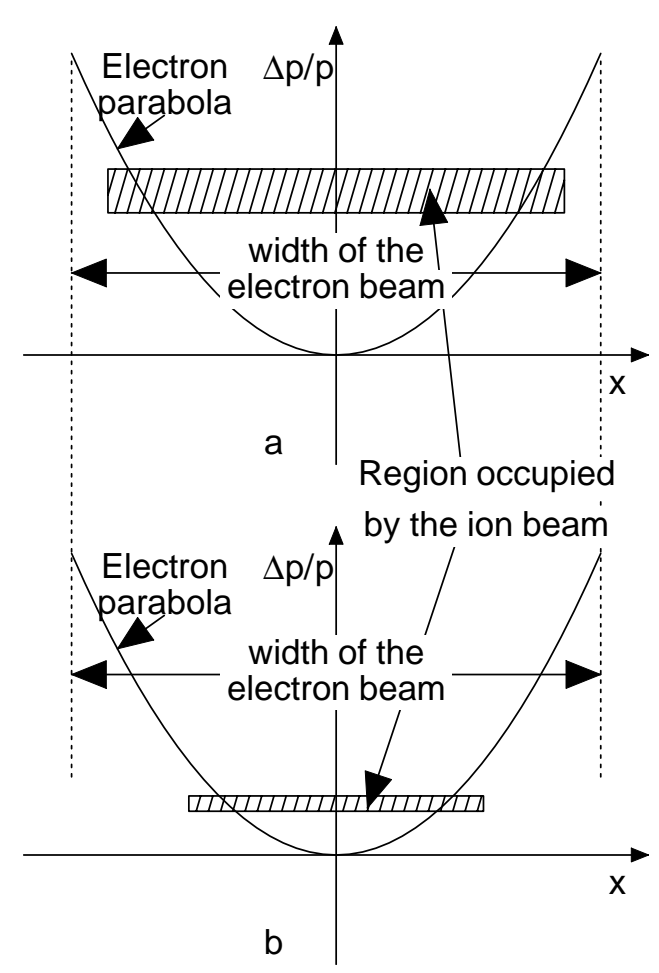

Fig. 4. Velocity versus position profile of the electron beam parabola and an ion beam with (a) large and (b) small betatron function at the location of the cooler.

\subsection{Increase in Cooling Rate due a Longer Cooler}

Precise alignment, in position and in angle, between the ion beam and the electron beam are mandatory for efficient electron cooling. Due to imperfections, the magnetic field lines guiding the electron cannot be perfectly straight. Thus the electron beam as a whole will follow a somehow curved trajectory. This makes it impossible to achieve an optimal positioning of the ion beam inside the electron parabola along the entire cooler and may reduce the cooling rate. For longer cooler length, the lateral offset between ion beam and the center of the electron is larger due to a larger lever arm. Thus, the reduction in cooling rate due to imperfect straightness of the magnetic field lines is stronger for a long cooler and high cooler currents. This may explain why doubling the cooler length led to faster cooling only for small cooler currents.

\section{Conclusions}

Qualitative arguments are given in an attempt to explain the experimentally observed dependence of cooling times on lattice parameters and cooler length. A key ingredient for all explanations is the parabolic dependence of the longitudinal velocity of the electrons on the transverse position, due to the potential 
created by the charge of the electron beam. Two different mechanisms are given that may explain the beneficial effect of finite dispersion on cooling.

For quantitative investigations, tracking with realistic models for the friction force, experienced by the ion beam in the cooler, are a prerequisite. This would allow as well to determine which of the two mechanisms, put forward to explain the beneficial effect of a dispersion on cooling, predominates. Moreover, better quantitative understanding of the observed dependence of electron cooling on lattice parameters would allow to optimize ion accumulation schemes.

In the framework of the LEIR project, a special multiturn injection (6) (with stacking in momentum and in betatron phase space) yields an injected beam with a large momentum spread on top of large emittances. This beam must be cooled into the stack. Thus the experimental results reported in section 2 cannot directly applied, since they were obtained with beams with a smaller momentum spread. However, from accumulation tests with large momentum spread beams, one can extrapolate that the required LEIR performance will be achieved with the design parameters, i.e. moderate betatron functions $\left(\beta_{T} \approx 5 \mathrm{~m}\right)$ and no dispersion at the cooler. This choice does not necessarily yield the fastest stacking rates, but the given LEIR geometry and hardware allows to set a small dispersion (up to about $|D| \approx 2 \mathrm{~m}$ ) at the cooler.

\section{References}

[1] D. Möhl, P. Lefèvre, A low Energy Accumulation Ring of Ions for LHC, CERN/PS 93-62 (DI).

[2] J. Bosser, C. Carli, M. Chanel, C. Hill, A. Lombardi, R. Maccaferri, S. Maury, D. Möhl, G. Molinari, S. Rossi, E. Tanke, G. Tranquille, M. Vretenar, Experimental Investigation of Electron Cooling and Stacking of Lead Ions in a Low Energy Accumulation Ring, Part. Acc. 63 (1999) pp. 171-210 and CERN/PS 99-033 (DI).

[3] J. Bosser, C. Carli, M. Chanel, L. Marie, D. Möhl, G. Tranquille, On the optimum dispersion of a storage ring for electron cooling with high space charge, Nucl. Instr. and Meth. A 441 (2000) 60-63.

[4] M. Beutelspacher, M. Grieser, D. Schwalm, A. Wolf, Dispersive Electron Cooling at the Heidelberg Heavy Ion Storage Ring TSR, Proc. of EPAC 2000 (Vienna).

[5] G. Tranquille, Optimum Parameters for Electron Cooling, Proc. of the Workshop on Beam Cooling and Related Topics COOL01 (Bad Honnef).

[6] M. Chanel, LEIR: the Low Energy Ion Ring at CERN, these Proceedings. 